Pacific

Journal of

Mathematics

SOME CHARACTERIZATION, UNIQUENESS AND EXISTENCE RESULTS FOR EUCLIDEAN GRAPHS OF CONSTANT MEAN CURVATURE WITH PLANAR BOUNDARY

JAIME RIPOLL 


\title{
SOME CHARACTERIZATION, UNIQUENESS AND EXISTENCE RESULTS FOR EUCLIDEAN GRAPHS OF CONSTANT MEAN CURVATURE WITH PLANAR BOUNDARY
}

\author{
JAIME RIPOLL
}

\begin{abstract}
We establish the existence and uniqueness of solutions to the Dirichlet problem for the cmc surface equation, including the minimal one, for zero boundary data, in certain domains of the plane. We obtain results that characterize the sphere and cmc graphs among compact embedded cmc surfaces with planar boundary satisfying certain geometric conditions. We also find conditions that imply that a compact embedded cmc surface which is a graph near the boundary is indeed a global graph.
\end{abstract}

\section{Introduction.}

In this paper we shall obtain some characterization, uniqueness and existence theorems to the Dirichlet's problem for the constant mean curvature $(\mathrm{cmc})$ equation with vanishing boundary data

$$
Q_{H}(u):=\operatorname{div} \frac{\nabla u}{\sqrt{1+|\nabla u|^{2}}}+2 H=0,\left.\quad u\right|_{\partial \Omega}=0, u \in C^{2}(\Omega) \cap C^{0}(\bar{\Omega})
$$

where $\Omega$ is a domain in the plane and $H \geq 0$. Although being a very special case of boundary data, it is shown in $[\mathbf{R}]$ that the general case of arbitrary continuous boundary data can be reduced, in many situations, to the zero boundary data.

In the minimal case, it is well known that if $\Omega$ is bounded and convex then there is a solution to $Q_{0}=0$ in $\Omega$ which assumes any continuous prescribed data value at $\partial \Omega$. For $\Omega$ convex but not bounded, and for some special cases of non-convex unbounded domains, R. Earp and H. Rosenberg ([ER]) proved the existence of solutions which take on any continuous bounded boundary value if $\Omega$ is not a half plane. This last case was treated by $\mathrm{P}$. Collin and R. Krust $([\mathbf{C K}])$ who proved existence for a given continuous boundary data with growth at most linear. In $[\mathbf{C K}]$ a uniqueness theorem in arbitrary unbounded domains is also obtained. 
Existence results in unbounded but not convex domains have been treated, starting with Nitsche $([\mathbf{N}])$ and more recently in $[\mathbf{E T}],[\mathbf{K T}]$ and $[\mathbf{R T}]$, in the so-called (finite) exterior domains, that is, domains $\Omega$ such that $\mathbb{R}^{2} \backslash \Omega$ is a (finite) union of pairwise disjoint bounded closed simply connected domains, and one can note a drastic difference between this case and the convex one. This can be seen in the non-existence theorem proved by N. Kutev and F. Tomi in $[\mathbf{K T}]$ : There are continuous necessarily non-zero boundary data $f$ on a given finite exterior domain, with arbitrarily small $C^{0}$ norm, for which no solution taking on the value $f$ on $\partial \Omega$ can exist. On the other hand, if one cuts a catenoid or, more generally, an embedded end of a minimal surface with finite total curvature by a plane, we get examples of minimal graphs on exterior of a closed curve, vanishing at the curve. This shows that one could expect the existence of solutions in finite exterior domains at least for special boundary data, for instance, zero boundary data. In fact, it is also proved in $[\mathbf{K T}]$ the existence of a solution in a finite exterior $C^{2, \alpha}$ domain for a generically small continuous boundary data $f$, that is, $f$ small in terms of bounds depending on the geometry of the boundary (see Theorem E in $[\mathbf{K T}])$. Still, it subsisted the question of existence of minimal graphs on arbitrary (finite or not) exterior $C^{0}$ domains even for zero boundary data. We answer here this question positively requiring a "periodicity" of the domain when it is not finite. Precisely:

Theorem 1. Let $\Gamma$ be a subgroup of the isometry group of $\mathbb{R}^{2}$ acting properly discontinuously in $\mathbb{R}^{2}$, and let $D$ be a fundamental domain of $\Gamma$. Let $\gamma_{1}, \ldots, \gamma_{m}$ be Jordan curves bounding closed domains $G_{i} \subset D, i=1, \ldots, m$ such that $G_{i} \cap G_{j}=\emptyset$ if $i \neq j$. Set

$$
\Omega=\mathbb{R}^{2} \backslash \bigcup_{\phi \in \Gamma} \phi\left(G_{1} \cup \ldots \cup G_{m}\right)
$$

and let $s \geq 0$ be given. Then there is a non-negative function $u_{s} \in C^{2}(\Omega) \cap$ $C^{0}(\bar{\Omega})$ solving (1) with $H=0$ in $\Omega$ such that

$$
\sup _{\Omega}\left|\nabla u_{s}\right|=s \text {. }
$$

It follows that

$$
\lim _{R \rightarrow \infty} \sup \frac{\max _{C_{R}} u_{s}}{R}<+\infty
$$

where $C_{R}$ is the circle of radius $R$ centered at the origin.

Concerning the above theorem we note that the technique of previous works ([N], $[\mathbf{E T}],[\mathbf{K T}],[\mathbf{R T}])$, of using catenoids as supersolutions, produces solutions having necessarily logarithm growth at infinity. Therefore, since in infinite exterior domains there are solutions with linear growth at infinity (Scherk's second minimal surface for example), it seems that this 
technique can not be used for proving the existence of solutions in this case. Concerning the growth of a minimal graph at infinity, we recall that Collin and Krust $([\mathbf{C K}])$ proved that

$$
\lim _{R \rightarrow \infty} \inf \left(\max _{C_{R}} u / \ln R\right)>0,
$$

where $u \neq 0$ is any non-negative solution of the minimal surface equation defined in a planar unbounded domain and vanishing at the boundary of the domain. The catenoid $v=\cosh ^{-1}(|x|)$ also shows that this estimate is optimal in the sense that

$$
\lim _{R \rightarrow \infty} \sup \frac{\max _{C_{R}} v}{\ln R}<+\infty .
$$

We remark that for the graphs constructed in Theorem 1 we can not, in general, improve (3) to (5), as one sees with the example of the second Sherck's minimal surface.

Considering now the case $H>0$, we recall the well-known result of J. Serrin stating that if $\Omega$ is bounded and $C^{2, \alpha}, 0<\alpha<1$, then (1) has an unique solution provided that $\partial \Omega$ has plane curvature bigger than or equal to $2 H$ (Theorem 1 of $[\mathbf{S}]$ ) (in fact, under this hypothesis, Serrin's theorem asserts that the Dirichlet problem for the mean curvature equation is uniquely solvable for any continuous (not necessarily zero) boundary data). As far as we know, there are no existence results for domains which are not simply connected, or even simply connected but not convex. Examples given by pieces of Delaunay surfaces however (see Proposition 1), show that we could expect the existence of cmc graphs over domains which are not simply connected, at least for the especial case of zero boundary data. In fact, we were able to obtain here an existence theorem for arbitrary (bounded) domains, with zero boundary data, but assuming also some restrictions on the geometry of the domains (see Theorem 2 below for the general statement). As a corollary of this theorem, we obtain:

Corollary 1. Let $\gamma, \gamma_{1}, \ldots, \gamma_{k}$ be $C^{2, \alpha}$ convex curves bounding closed domains $E, E_{1}, \ldots, E_{k}$ such that $E_{i} \subset E \backslash \partial E, E_{i} \cap E_{j}=\emptyset$ if $i \neq j, 0<\alpha<1$. Given $H>0$, we require that the curvatures $\kappa$ and $\kappa_{i}$ of $\gamma$ and $\gamma_{i}$ satisfy

$$
3 H \leq a<\kappa<\kappa_{i}<a\left(\frac{a}{H}-2\right), \quad i=1, \ldots, k
$$

for some $a>0$. Then there exists a solution to (1) in $\Omega:=E \backslash\left(\cup_{i=1}^{k} E_{i}\right)$ belonging to $C^{2, a}(\bar{\Omega})$.

In the next results we study in more details the Dirichlet problem in convex domains of the plane. Recently, Rafael López and Sebastián Montiel proved the existence of a solution of (1) on a convex domain $\Omega$ provided that the length $L$ of $\partial \Omega$ satisfies $L H \leq \sqrt{3} \pi$ (Corollary 4 of $[\mathbf{L M}]$ ). It follows from this result that if the curvature $k$ of $\partial \Omega$ satisfies $k>(2 / \sqrt{3}) H$ then 
(1) has a solution (see the remark after the proof of Corollary 4 of $[\mathbf{L M}]$ ), improving Serrin's theorem for zero boundary data. This same conclusion had already been obtained by L.E. Payne and G.A. Philippin in $[\mathbf{P P}]$ (see Theorem 5, Equation 3.11 of $[\mathbf{P P}]$ ). As a corollary of our Theorem 2, we obtain the optimal estimate of the lower bound for the curvature, namely:

Corollary 2. If $\Omega$ is convex, bounded and $C^{2, \alpha}$, (1) is solvable provided that the curvature $k$ of $\partial \Omega$ satisfies $k \geq H$. Furthermore, if $k>H$ then the solution belongs to $C^{2, \alpha}(\bar{\Omega})$.

The above improvement is optimal in the sense that given any $0<\epsilon<1$, the curvature $k$ of a disk of radius $\epsilon H$ satisfies $k \geq \epsilon H$ but there is no solution to $Q_{H}=0$ (with any boundary value) in this disk.

We obtained the following apparently technical result but which seems to be useful for applications:

Theorem 3. Let $H>0$ be given and let $\Omega$ be a $C^{2, \alpha}$ bounded convex domain, $0<\alpha<1$, satisfying the following condition: There exists a $<1 /(2 H)$ such that, given $h \in[0, H]$, any solution $u \in C^{2}(\bar{\Omega})$ to $Q_{h}=0$ in $\Omega$ with $\left.u\right|_{\partial \Omega}=0$ satisfies the a priori height estimate $|u| \leq a<1 /(2 H)$. Then there is a solution $u \in C^{2, \alpha}(\bar{\Omega})$ to $Q_{H}=0$ in $\Omega$ with $\left.u\right|_{\partial \Omega}=0$.

As a corollary of Theorem 3, we obtain a result that extends Corollary 2 above and Corollary 4 of $[\mathbf{L M}]$ in other directions.

Corollary 3. Let $H>0$ be given and let $\Omega$ be a convex domain contained between two parallel lines $1 / H$ far apart. Then (1) is solvable in $\Omega$.

We observe that none two of these results, namely, Corollaries 2 and 3 above and Corollary 4 of $[\mathbf{L M}]$ are comparable.

It is proved in [EFR], Corollary 5, that any unbounded convex domain admitting a bounded solution to (1) is necessarily contained between two parallel lines $1 / H$ far apart. Using this result and Corollary 3 above, we obtain:

Corollary 4. Let $\Omega$ be a convex unbounded domain. Then (1) is solvable in $\Omega$ if and only if $\Omega$ is contained between two parallel lines $1 / H$ far apart.

One can also use Theorem 3 to prove the existence of solutions to the Dirichlet problem with hypothesis on the area do the domain. For doing this, it is necessary to obtain an estimate of the area of the domain in terms of the height of the graph, what is done in the next result.

Proposition 2. Let $\Omega$ be a bounded domain in the plane and let $u \in C^{2}(\Omega) \cap$ $C^{0}(\bar{\Omega})$ be a non-negative solution to $Q_{H}=0$ in $\Omega$ with $\left.u\right|_{\partial \Omega}=0$. Let $G$ be the graph of $u$ and set $h=\max _{\Omega} u$. Then

$$
\text { Area }(G)<(1+2 h H) \operatorname{Area}(\Omega)
$$


and

$$
\frac{2 \pi h}{H(1+2 h H)}<\operatorname{Area}(\Omega)
$$

Corollary 5. Let $\Omega$ be a bounded convex domain in the plane such that 2 Area $(\Omega) H^{2}<\pi$. Then there is a solution of (1) in $\Omega$.

Corollary 3 implies that one has examples of bounded convex domains with arbitrarily large area (and therefore perimeter) where (1) has a solution. Therefore, there is no chance to get a converse on either Corollary 5 above or Corollary 4 of $[\mathbf{L M}]$ : The existence of a solution of (1) in a convex bounded domain $\Omega$ does not imply the existence of an upper bound for Area $(\Omega) H^{2}$ (or $L(\Omega) H$ ). Nevertheless, we believe that Corollary 5 is not optimal. It implies the a priori height $1 /(2 H)$ for the cmc $H$ graphs on $\Omega$ which seems to be too "low". We might expect that Area $(\Omega) H^{2}<\pi$ should enough to guarantee the solvability of (1) in $\Omega$.

In the last years a number of papers have been written studying compact cmc surfaces whose boundary is a given Jordan curve in $\mathbb{R}^{3}$. Many related problems however remain still opened. For example, it is not known if an embedded cmc surface in $\mathbb{R}_{+}^{3}=\{z \geq 0\}$ whose boundary is a convex curve in the plane $P=\{z=0\}$ can have genus bigger than 0 (see $[\mathbf{R R}]$ and references therein). Using our previous results, we were able to prove that if the slope of the tangent planes of $M$ at $\partial M$ are not too small (in terms of the boundary data), then the surface is either a graph or part of a sphere. In particular, the surface is a topological disk. Precisely, we prove:

Theorem 4. Let $M$ be an embedded connected cmc $H>0$ surface with boundary $\partial M$ in the plane $P=\{z=0\}$. We assume that $M$ is contained in the half space $z \geq 0$ and that any connected component of $\partial M$ is a convex curve. Assume furthermore that the closed interior of any two curves in $\partial M$ have disjoint intersection. Set $N=(1 / H) \vec{H}$, where $\vec{H}$ is the mean curvature vector of $M$, and let $n$ denote the unit vector along $\partial M$ in the plane $P$ normal to $\partial M$ and pointing to the bounded connected components of $P \backslash \partial M$.

Given any connected component $C$ of $\partial M$, if one of the alternatives below holds, then $M$ is either a graph over $P$ or a part of sphere of cmc H. In particular, the genus of $M$ is zero.

(a) Setting $\kappa_{0}=\min \kappa_{C}$, where $\kappa_{C}$ is the curvature of $C$ in the plane $P$, we require that $\kappa_{0} \geq H$ and $\langle N(p), n(p)\rangle \geq H / \kappa_{0}$ if $\left\langle N(p), e_{3}\right\rangle \geq 0$, $p \in C\left(e_{3}=(0,0,1)\right)$.

(b) Setting $d=\inf d\left(l_{1}, l_{2}\right)$, where $l_{1}, l_{2}$ are any two parallel lines in $P$ such that $C$ is in between $l_{1}$ and $l_{2}$, and $d\left(l_{1}, l_{2}\right)=\inf \left\{\|p-q\|, p \in l_{1}\right.$, 
$\left.q \in l_{2}\right\}$, we require that $d H \leq 1$, and

$$
\langle N(p), n(p)\rangle \geq \frac{d H}{\sqrt{1-d^{2} H^{2}}}
$$

if $\left\langle N(p), e_{3}\right\rangle \geq 0, p \in C$.

(c) Denoting by $A$ the area of the region enclosed by $C$, we require that $2 A H^{2}<\pi$ and

$$
\begin{aligned}
&\langle N(p), n(p)\rangle \geq \frac{H \sqrt{A\left(2 \pi-3 H^{2} A\right)}}{\pi-2 H^{2} A} \\
& \text { if }\left\langle N(p), e_{3}\right\rangle \geq 0, p \in C .
\end{aligned}
$$

R. López and S. Montiel proved that an embedded compact cmc surface $M$ in $\mathbb{R}^{3}$ with area $A$ satisfying $A H^{2} \leq \pi$ is necessarily a graph ([LM]). The corollary of Theorem 4 that follows gives an additional characterization of a graph from a bound on the area of the surface, where the condition $A H^{2} \leq \pi$ is not necessarily satisfied. Just as an example, one may deduce from Corollary 6 below that if $H^{2} A \leq(95 / 84) \pi$ and $\partial M$ is a convex planar curve bounding a domain with area $a$ satisfying $(5 / 12) \pi \leq a H^{2} \leq \pi / 2$, then $M$ is a graph.

Corollary 6. Let $M$ be a compact embedded surface of cmc $H$ whose boundary $\partial M$ is a convex curve in the plane $z=0$, boundary of a planar domain $\Omega$. Denote by $A$ the area of $M$ and by a the area of $\Omega$. Assume that $2 a H^{2} \leq \pi$ and that

$$
A \leq \frac{2 \pi-a H^{2}}{\pi-a H^{2}} a
$$

Then $M$ is a graph.

Next, we consider a more general situation: The boundary $\partial M$ of $M$ (compact embedded cmc $H$ surface) is not necessarily plane but has a $1-1$ projection over a convex curve $\gamma$ in the plane $z=0$. By taking the right cylinder $C$ over $\gamma$, we assume that $M$ does not intersect the connected component of $C \backslash \partial M$ which is below $\partial M$. We prove that if there exists a neighborhood of $\partial M$ in $M$ which is a graph over a neighborhood of $\gamma$ in $\bar{\Omega}$, where $\Omega$ in the interior of $\gamma$, then $M$ is a graph over $\Omega$ (Theorem 5 ). These hypothesis can be weakened when $\partial M$ is a plane curve: If there is a neighborhood of $\partial M$ in $M$ contained in the half space $z \geq 0$ which is a graph over a neighborhood of $\partial M$ in $\bar{\Omega}$, then $M$ is a graph (Theorem 6).

\section{The minimal case.}

Proof of Theorem 1. If $s=0$ then Theorem 1 has a trivial proof. Thus, let us assume that $s>0$. We first consider the case that $\Omega$ is a $C^{\infty}$ domain. Given $n$ denote by $D_{n}^{1}$ the open disk centered at the origin with radius $n$ and 
assume that $n$ is such that $D_{n}^{1}$ contains $G_{1} \cup \ldots \cup G_{m}$. Let $E_{1}, \ldots, E_{k(n)}$ be the closed domains of $\mathbb{R}^{2} \backslash \Omega$ which are contained in $D_{n}^{1}$, set $a_{i}=\partial E_{i}$, $\alpha_{n}=a_{1} \cup \ldots \cup a_{k(n)}$.

Let $L_{n}$ be the catenoid tangent to the cylinder $H=C_{n}^{1} \times \mathbb{R}, C_{n}^{1}=\partial D_{n}^{1}$, along the circle $C_{n}^{1}$. Assume that $L_{n} \cap\{z \geq 0\}$ is the graph of the function $v_{n}$ in $\mathbb{R}^{2} \backslash D_{n}^{1}$. We choose $R_{n} \geq n^{2}$ such that $\left|\nabla v_{n}\right| \leq s / 2$ at the circle $C_{n}^{2}$ centered at the origin with radius $R_{n}$. Let $N$ be the unit normal vector to the catenoid that points to the rotational axis. Set $I_{n}=H_{n} \cap L_{n} \cap\{z \geq 0\}$, where $H_{n}:=C_{n}^{2} \times \mathbb{R}$. Set $\Omega_{n}=D_{n}^{2} \backslash\left(E_{1} \cup \ldots \cup E_{k(n)}\right)$, where $D_{n}^{2}$ is the disk bounded by $C_{n}^{2}$.

We set

$$
\begin{aligned}
T_{n} & =\left\{t \geq 0 \mid \exists u_{t} \in C^{\infty}\left(\overline{\Omega_{n}}\right), \text { such that } Q_{0}\left(u_{t}\right)=0,\right. \\
\sup _{\overline{\Omega_{n}}}\left|\nabla u_{t}\right| & \left.\leq s, \text { and }\left.u_{t}\right|_{\alpha_{n}}=0,\left.u_{t}\right|_{C_{n}^{2}}=t\right\} .
\end{aligned}
$$

We have $T_{n} \neq \emptyset$ since $0 \in T_{n}$ and obviously $\sup T_{n}<+\infty$. Set $t_{n}=\sup T_{n}$. We prove that $t_{n} \in T_{n}$ and that $\sup _{\alpha_{n}}\left|\nabla u_{t_{n}}\right|=s$. Given $t \in T_{n}$, we first observe that $\sup _{C_{n}^{2}}\left|\nabla u_{t}\right| \leq s / 2$. In fact: Let $\eta$ denote the interior unit normal vector to $C_{n}^{2}$, and denote also by $\eta$ its extension to $\mathbb{R}^{3} \backslash\{z-$ axis $\}$ by radial translation in each plane $z=c$, and let $N_{t}$ be the unit normal vector to the graph $G_{t}$ of $u_{t}$ pointing upwards. Since $G_{t}$ is contained in the convex hull of its boundary, we have $\left\langle N_{t}, \eta\right\rangle \geq 0$ at $I_{n}$.

Moving $L_{n}$ down if necessary, we have that $L_{n} \cap G_{t}=\emptyset$. Going up with $L_{n}$ until it touches the circle $C_{n, t}^{2}$ centered at the $z$-axis, with radius $R_{n}$, contained in the plane $z=t$, we will obtain, from the maximum principle, that $\left\langle N_{t}, \eta\right\rangle\left\langle\langle N, \eta\rangle<1\right.$ at $I_{n}$ (recall that any vertical translation of $L_{n}$ does not intersect any curve $\alpha_{i}$ ). Since, by construction, $L_{n}$ is given as a graph of a function $v_{n}$ such that $\left|\nabla v_{n}\right| \leq s / 2$ at $C_{n}^{2}$ it follows that $\left|\nabla u_{t}\right| \leq s / 2$ at $C_{n}^{2}$.

Let $\left\{s_{m}\right\} \subset T_{n}$ be a sequence converging to $t_{n}$ as $m \rightarrow \infty$. Since the functions $u_{s_{m}}$ are uniformly bounded having uniformly bounded gradient, standard $C^{k}$ estimates guarantee us the existence of a subsequence of $\left\{u_{s_{m}}\right\}$ converging uniformly $C^{\infty}$ on $\overline{\Omega_{n}}$ and to a solution $w \in C^{\infty}\left(\overline{\Omega_{n}}\right)$ of $Q_{0}=0$ in $\Omega_{n}$. One of course has $\sup _{\bar{\Omega}_{n}}|\nabla w| \leq s,\left.w\right|_{C_{n}^{2}}=t_{n}$ and $\left.w\right|_{\alpha_{n}}=0$, so that $t_{n} \in T_{n}$ and $w=u_{t_{n}}$.

Suppose that $\sup _{\overline{\Omega_{n}}}\left|\nabla u_{t_{n}}\right|<s$. Applying the implicit function theorem, one can guarantee the existence of a solution $u^{\prime} \in C^{\infty}\left(\overline{\Omega_{n}}\right)$ to $Q_{0}=0$ vanishing at $\alpha_{n}$ and taking on a value $t_{n}+\epsilon$ on $C_{n}^{2}, \epsilon>0$. For $\epsilon$ small enough, one still has $\sup _{\overline{\Omega_{n}}}\left|\nabla u^{\prime}\right|<s$. It follows that $t_{n}+\epsilon \in T$, a contradiction! Therefore, $\sup _{\overline{\Omega_{n}}}\left|\nabla u_{t_{n}}\right|=s$. Since $\sup _{C_{n}^{2}}\left|\nabla u_{t_{n}}\right| \leq s / 2$, we obtain, from the gradient maximum principle, $\sup _{\alpha_{n}}\left|\nabla u_{t_{n}}\right|=s$. 
We define now a sequence $\left\{u_{n}\right\}$ of non-negative solutions to $Q_{0}=0$ in the domain $\Lambda_{n}:=D_{n}^{3} \cap \Omega$, where $D_{n}^{3}$ is an open disk centered at the origin with radius $n^{2}-n$, such that $\left.u_{n}\right|_{\partial \Lambda_{n} \backslash \partial D_{n}^{3}}=0$ and

$$
\sup _{\partial \Lambda_{n} \cap D}\left|\nabla u_{n}\right|=\sup _{D_{n}^{3}}\left|\nabla u_{n}\right|=s
$$

if $D_{n}^{3}$ contains $D$, as follows. Given $n$ such that the disk $D_{n}^{1}$ contains the fundamental domain $D$, from what we have proved above there exists $p_{n} \in$ $\alpha_{n}$ such that $\left|\nabla u_{t_{n}}\left(p_{n}\right)\right|=s$.

If $p_{n} \in D$, then we set $u_{n}=\left.u_{t_{n}}\right|_{\Lambda_{n}}$. If $p_{n} \notin D$, we take an isometry $\phi \in \Gamma$ such that $\phi\left(p_{n}\right) \in D$, and define $u_{n}(p)=u_{t_{n}}\left(\phi^{-1}(p)\right)$, for $p \in \Lambda_{n}$. Since $\left|p_{n}\right|<n$, it follows $\phi^{-1}(p) \in D_{n}^{2}$, for all $p \in D_{n}^{3}$, so that $u_{n}$ is well defined and satisfies the stated conditions.

By standard $C^{k}$ estimates, it follows that the sequence $\left\{u_{n}\right\}$ contains a subsequence that converges uniformly on compacts of $\bar{\Omega}$ to a solution $u \in C^{\infty}(\bar{\Omega})$ to $Q_{0}=0$ such that $\left.u\right|_{\partial \Omega}=0$ and (2) is obviously satisfied.

If $\Omega$ is just $C^{0}$, we can take sequences $\gamma_{i, n}$ of $C^{\infty}$ curves contained in $G_{i}$, for each $1 \leq i \leq m$ such that $\gamma_{i, n}$ converges $C^{0}$ to $\gamma_{i}$ as $n \rightarrow \infty$. We can therefore apply the result obtained above and compactness results to guarantee the existence of a solution of (1) in $\Omega$, concluding the proof of Theorem 1.

Remark. The proof of Theorem 1 is "experimental" in the sense that one can reproduce the arguments of the proof by using soap films. To obtain experimentally a solution $u_{t_{n}}$ of $Q_{0}=0$ in $\Omega_{n}$, we represent the curves $a_{1}, \ldots, a_{n}$ and the circle $C_{n}^{2}$ by wires and embed them in a soaped water, taking care that they are kept in the same plane. Then take them out from the water and drill the soap films that are enclosed by the wires $a_{1}, \ldots, a_{n}$ : One obtains the zero solution in $\Omega_{n}$. Now, given $s>0$, we lift the circle $C_{n}^{2}$ up until the soap film reaches the slope $s$ at some curve $a_{i}$. The resulting soap film is the graph of $u_{t_{n}}$.

\section{The case $H>0$.}

In order to state and prove some of the next results, we need to introduce some notations and definitions.

Consider a bounded open domain $\Omega$ in the plane whose boundary consists of a finite number of $C^{2}$ embedded Jordan curves. Set $\Omega^{c}=\mathbb{R}^{2} \backslash \Omega$ and let $p \in \partial \Omega$ be given. If $\Omega$ is globally convex at $p$, let $l_{1}(p, \Omega)$ be the tangent line to $\partial \Omega$ at $p$ and let $l_{2}(p, \Omega)$ be the closest parallel line to $l_{1}(p, \Omega)$ such that $\Omega$ is between $l_{1}(p, \Omega)$ and $l_{2}(p, \Omega)$. We then set

$$
R_{1}(p, \Omega)=d\left(l_{1}(p, \Omega), l_{2}(p, \Omega)\right)=\inf \left\{\left|q_{1}-q_{2}\right| \mid q_{i} \in l_{i}(p, \Omega), i=1,2\right\} .
$$

If $\Omega$ is not globally convex at $p$, denote by $C_{1}(p, \Omega)$ the circle tangent to $\partial \Omega$ at $p$, contained in $\Omega^{c}$, and whose radius is the biggest one among those 
circles satisfying these properties. Denote by $C_{2}(p, \Omega)$ the circle with the same center as $C_{1}(p, \Omega)$ of smallest radius $R_{2}(p, \Omega)$ containing $\Omega$.

If $\partial \Omega$ admits a circle tangent to $\Omega$ at $p$ and containing $\Omega$ in its interior, let $R_{3}(p, \Omega)$ be the smallest radi of these circles. If $\Omega$ does not admit such a circle at $p$, we set $R_{3}(p, \Omega)=\infty$.

Finally, set

$$
W(p, \Omega)=\min \left\{R_{1}(p, \Omega), R_{2}(p, \Omega), R_{3}(p, \Omega)\right\} .
$$

Theorem 2. Let $H_{0}>0$ be given and let $\Omega$ be a $C^{2, \alpha}$ bounded domain in the plane $z=0$. Given a point $p$ of $\partial \Omega$, let $r(p, \Omega), 0<r(p, \Omega) \leq \infty$, be the radius of the circle $C_{1}(p, \Omega)$ (the circle of biggest radius tangent to $\partial \Omega$ at $p$ and contained in $\left.\mathbb{R}^{2} \backslash \Omega\right)$. We require that

$$
W(p, \Omega) \leq \frac{2}{H_{0}\left(1+\sqrt{1+\frac{1}{r(p, \Omega) H_{0}}}\right)}
$$

for all $p \in \partial \Omega$. Then the Dirichlet problem (1) is solvable for any $0 \leq H \leq$ $H_{0}$. Furthermore, if the inequality is strict in (7), for all $p \in \partial \Omega$, then the solution is in $C^{2, \alpha}(\bar{\Omega})$.

For proving Theorem 2 we will use, as barriers, rotational graphs with cmc described in the proposition below.

Proposition 1. Let $0<r$ and $H>0$ be given. Then there exists a rotational graph with cmc $H$ defined on an annulus in the plane whose boundary consists of two concentric circles of radii $r$ and $R$, where $R$ satisfies:

$$
R \geq \frac{2}{H\left(1+\sqrt{1+\frac{1}{r H}}\right)} .
$$

Proof. It is well-known that there is a nodoid $N$ in the plane $x-z$ generating, by rotation around the $z$-axis, a cmc $H$ surface (with self intersections) whose distance to the rotational axis is $r$. We can assume that the point $A=(r, 0)$ belongs to $N$. We consider an embedded piece $N_{r}$ of $N$ from the point $A$ to the closest point $B$ of self intersection of $N$ and such that the $z$-coordinate of any point of $N_{r}$ is non-negative. The coordinates of $B$ are of the form $(0, R), R>r$.

The rotation of $N_{r}$ around the $z$-axis is a graph over the plane $z=0$ which vanishes along two circles centered at the origin with radius $r$ and $R$, being orthogonal to the circle of radius $r$. We prove that $R$ satisfies (8).

It is known that if $x=x(t), z=z(t)$ represent a piece of a generating curve of a cmc $H$ rotational surface, then these functions satisfy the first order system of ordinary differential equations (see Lemma 3.15 of [doCD]):

$$
\left\{\begin{array}{l}
\left(\frac{d x}{d t}\right)^{2}=1-\left(H x-\frac{a}{x}\right)^{2} \\
\left(\frac{d x}{d t}\right)^{2}+\left(\frac{d z}{d t}\right)^{2}=1
\end{array}\right.
$$


where $a$ is a constant. In the points where $x=x(z)$, we therefore get

$$
x^{\prime 2}=\left(\frac{x}{a-H x^{2}}\right)^{2}-1 .
$$

We then have

$$
z^{\prime}(x)=\frac{1}{\sqrt{\left(\frac{x}{a-H x^{2}}\right)^{2}-1}}
$$

for $r \leq x \leq x_{1}$, and

$$
z^{\prime}(x)=-\frac{1}{\sqrt{\left(\frac{x}{a-H x^{2}}\right)^{2}-1}}
$$

for $x_{1} \leq x \leq R$, where $x_{1} \in(r, R)$ is such that $z^{\prime}\left(x_{1}\right)=0$. Since $z^{\prime}(r)=\infty$, we have

$$
\left(\frac{r}{a-H r^{2}}\right)^{2}-1=0
$$

and $a=r(-1+H r)$ or $a=r(1+H r)$. In the case of the nodoids, we know moreover that $x^{\prime}\left(z_{1}\right)=\infty$, where $z_{1}=z\left(x_{1}\right)$. Therefore we have $a=H x_{1}^{2} \geq H r^{2}$, and this implies that $a=r(1+r H)$. It follows that

$$
x_{1}=\sqrt{\frac{r(1+r H)}{H}} .
$$

Now, a computation shows that

$$
z^{\prime}\left(x_{1}-x\right) \geq-z^{\prime}\left(x_{1}+x\right),
$$

for all $x \in\left[0, x_{1}-r\right]$. It follows then that

$$
R \geq x_{1}+\left(x_{1}-r\right) \geq \frac{2}{H\left(1+\sqrt{1+\frac{1}{r H}}\right)}
$$

proving the proposition.

Proof of Theorem 2. Considering the family of Dirichlet's problems

$$
\operatorname{div} \frac{\nabla u}{\sqrt{1+|\nabla u|^{2}}}=-2 h,\left.\quad u\right|_{\partial \Omega}=0, u \in C^{2}(\bar{\Omega}), h \in\left[0, H_{0}\right)
$$

we first prove that

$$
S:=\left\{h \in\left[0, H_{0}\right) \mid(9) \text { has a solution }\right\}
$$

coincides with $\left[0, H_{0}\right)$. Since (9) has the trivial solution for $h=0$, we have $S \neq \emptyset$. From the implicit function theorem, it follows that $S$ is open in $\left[0, H_{0}\right)$. For proving that $S$ is closed in $\left[0, H_{0}\right)$, let us consider a sequence $\left\{h_{n}\right\} \subset S$ converging to $H_{1} \in\left[0, H_{0}\right)$. If $H_{1}=0$ then $H_{1} \in S$ and we are done. Thus, let us assume that $H_{1}>0$. By contradiction, assume that 
$H_{1} \notin S$. Given $n$, let $u_{n} \in C^{2}(\bar{\Omega})$ be a solution of (9) for $h=h_{n}$. We claim that the gradient of the solutions $u_{n}$ can not be uniformly bounded. In fact: Otherwise, since by very known height estimates, $u_{n}$ satisfies

$$
\left|u_{n}\right| \leq \frac{1}{H_{1}-\epsilon}
$$

for all $n$ bigger than a certain $n_{0}$, for some $\epsilon>0$ smaller than $H_{1}$ and, by assumption, the gradient of the $u_{n}$ are uniformly bounded, using standard $C^{k}$ estimates, $k \geq 2$, one can extract a subsequence of $u_{n}$ converging uniformly on compacts of $\Omega$ to a solution $u \in C^{\infty}(\Omega) \cap C^{0}(\bar{\Omega})$ of $Q_{H_{1}}=0$, such that $\left.u\right|_{\partial \Omega}=0$. Since $\Omega$ is a $C^{2, \alpha}$ domain, $u$ is $C^{\infty}$ in $\Omega$ and has bounded gradient, $u$ extends $C^{2, \alpha}$ to $\bar{\Omega}$ (this is well known. It can be proved by reducing the problem to linear uniformly elliptic operators and applying standard arguments: See p. 249 paragraph 3 and Remark 1 p. 253 of $[\mathbf{G T}]$. See also Lemma 1.1 of $[\mathbf{E F R}])$. Therefore $u$ solves (9) for $h=H_{1}$, implying that $H_{1} \in S$, a contradiction! This proves our claim.

Therefore, there is a sequence $p_{n} \in \partial \Omega$ such that $\left|\nabla u_{n}\left(p_{n}\right)\right| \rightarrow \infty$, as $n \rightarrow \infty$. Without loss of generality, we may assume that $p_{n}$ converges to $p \in \partial \Omega$. Denote by $G_{n}$ the graph of $u_{n}$. Since $H_{1}<H_{0}$, condition (7) allows us to take $H^{\prime} \in\left(H_{1}, H_{0}\right)$ and $0<r^{\prime}<r(p, \gamma)$ such that

$$
W(p, \Omega)<\frac{2}{H^{\prime}\left(1+\sqrt{1+\frac{1}{r^{\prime} H^{\prime}}}\right)} .
$$

According to our previous notations, if $W(p, \Omega)=R_{3}(p, \Omega)$, then $\bar{\Omega}$ is contained in an open disk $D$ of radius $1 / H^{\prime}$, since then

$$
R_{3}(p, \Omega)<\frac{2}{H^{\prime}\left(1+\sqrt{1+\frac{1}{r^{\prime} H^{\prime}}}\right)}<\frac{1}{H^{\prime}} .
$$

If $W(p, \Omega)=R_{1}(p, \Omega)$, then $\bar{\Omega}$ is contained in an open strip $S$ having as boundary two parallel lines $1 / H^{\prime}$ far apart, since in this case there holds (10) with $R_{1}(p, \Omega)$ in place of $R_{3}(p, \Omega)$. Finally, in the case that $W(p, \Omega)=$ $R_{2}(p, \Omega)$ we conclude that $\bar{\Omega}$ is contained in an annulus $A$ of radius $r^{\prime}$ and $R^{\prime}:=R_{2}(p, \Omega)$ with

$$
R^{\prime}<\frac{2}{H^{\prime}\left(1+\sqrt{1+\frac{1}{r^{\prime} H^{\prime}}}\right)} .
$$

In this last case, we can use Proposition 1 to guarantee the existence of a graph $G$ of $\mathrm{cmc} H^{\prime}$ (a piece of nodoid), defined in an annulus $A^{\prime}$ with $A \subset A^{\prime}$. Therefore, if $U^{\prime}$ denotes either $D, S$ or $A^{\prime}$, we may consider a cmc $H^{\prime}$ graph $G^{\prime}$ of a function $u^{\prime}$ defined in $U^{\prime}$ with $\bar{\Omega} \subset U^{\prime}$ such that $G^{\prime}$ is either a half sphere if $U^{\prime}=D$, a half cylinder if $U^{\prime}=S$ or $G^{\prime}=G$ if $U^{\prime}=A^{\prime}$. 
Moving $G^{\prime}$ slightly down in such a way that its domain $U^{\prime}$ still contains $\Omega$, we may assume that

$$
\left|\nabla u^{\prime}(p)\right|<C<\infty, p \in \partial U^{\prime} .
$$

Now, we can move $U^{\prime}$ towards $\Omega$ until the boundary of $U^{\prime}$ is tangent to $\gamma$ at $p$ and in such a way that $\partial U^{\prime}$ does not intersect $\partial \Omega$ along this motion. By choosing $n$ big enough, we have $\left|\nabla u_{n}\right|>C$ so that the graph $G_{n}$ of $u_{n}$ and $G^{\prime}$ intersect themselves in interior points. But then, moving $G_{n}$ vertically down until it reaches the last contact with $G^{\prime}$, we obtain a tangency between $G_{n}$ and $G^{\prime}$ in an interior point, with $G_{n}$ below $G^{\prime}$, a contradiction, since the mean curvature $h_{n}$ of $G_{n}$ satisfies $h_{n}<H^{\prime}$ for $n$ big enough. Therefore, $H_{1} \in S$, and this proves the existence of a solution for (9) for $H \in\left[0, H_{0}\right)$.

To prove the existence of a solution for $H=H_{0}$ of (1), let us consider a increasing sequence $H_{n}<H_{0}$ with $\lim _{n \rightarrow \infty} H_{n}=H_{0}$. Given $n$, let $u_{n}$ be a solution of (1) in $\Omega$ for $H=H_{n}$. By the maximum principle, the sequence $\left\{u_{n}\right\}$ is monotonically increasing, and standard compactness results guarantee us the $\left\{u_{n}\right\}$ converges uniformly on compacts of $\Omega$ to a solution $u \in C^{2}(\Omega)$ of $Q_{H}=0$ (given by $u(p)=\lim _{n} u_{n}(p), p \in \Omega$ ). To prove that $u \in C^{0}(\bar{\Omega})$, let us consider a sequence $p_{n} \in \Omega$ converging to $p \in \partial \Omega$. As done above, we can consider a solution $v \in C^{2}(\Lambda) \cap C^{0}(\bar{\Lambda})$ to $Q_{H}=0$ where $\Lambda$ is a domain containing $\Omega$ with $p \in \partial \Lambda$ and such that $\left.v\right|_{\partial \Lambda}=0$ (the graph of $v$ is either part of sphere, a cylinder, or a Delaunay surface, and the gradient of $v$ may have infinity norm at the boundary of the domain). By the maximum principle, $u_{m}\left(p_{n}\right) \leq v\left(p_{n}\right)$, for all $n$ and $m$. It follows that $0 \leq \lim _{n} u\left(p_{n}\right)=\lim _{n} \lim _{m} u_{m}\left(p_{n}\right) \leq \lim _{n} v\left(p_{n}\right)=0$. This proves that $u \in C^{2}(\Omega) \cap C^{0}(\bar{\Omega})$ and $\left.u\right|_{\partial \Omega}=0$, that is, $u$ solves (1) for $H=H_{0}$ in $\Omega$.

Now, if the inequality is strict in (7), we get a solution of (1) in $\Omega$ whose gradient has bounded norm in $\Omega$. Since $\Omega$ is $C^{2, \alpha}$, standard arguments already used above imply that the solution extends $C^{2, \alpha}$ to $\bar{\Omega}$, concluding the proof of Theorem 2.

Proof of Corollary 1. We have just to assure that $\Omega$ satisfies the hypothesis of Theorem 2. Given $p \in \partial \Omega$, if $p \in \gamma$ then $r(p, \Omega)=\infty$, so that we must have $W(p, \Omega)<1 / H$, what is obviously true since the curvature of $\gamma$ satisfies $\kappa \geq 3 H$. If $p \in \gamma_{i}$ for some $i$, then we can place a circle centered at $z(p, \Omega)$ of radius $r$ tangent $\gamma_{i}$ and contained in $E_{i}$, such that

$$
r>\frac{1}{a\left(\frac{a}{H}-2\right)} \text {. }
$$

It follows from this that

$$
\frac{2}{a}<\frac{2}{H\left(1+\sqrt{1+\frac{1}{r H}}\right)} .
$$


On the other hand, since the geodesic curvature $\kappa$ of $\gamma$ satisfies $\kappa>a, \Omega$ is contained in a circle of radius $2 / a$ centered at $z(p, \Omega)$, so that $W(p, \Omega)<2 / a$, showing that $(7)$ is satisfied and concluding the proof of the corollary.

Proof of Corollary 2. In the case that $\partial \Omega$ is a convex curve the second hand of (7) equals to $1 / H$ for all $p \in \partial \Omega$, since $r(p, \partial \Omega)=\infty$, for all $p \in \partial \Omega$. Therefore, if one requires that the curvature of $\partial \Omega$ is bigger than or equal to $H,(7)$ is everywhere satisfied, proving the corollary.

Proof of Theorem 3. Set

$$
T=\left\{h \in[0, H] \mid \exists u \in C^{2}(\bar{\Omega}) \text { such that } Q_{h}(u)=0 \text { in } \Omega,\left.u\right|_{\partial \Omega}=0\right\} .
$$

Then $0 \in T$ so that $T \neq \emptyset$. From the implicit function theorem, $T$ is open. Let $h_{n} \in T$ be a sequence converging to $h \in[0, H]$. Let $u_{n}$ be the solution to $Q_{h_{n}}=0$ in $\Omega$ such that $\left.u_{n}\right|_{\partial \Omega}=0, u_{n} \in C^{2}(\bar{\Omega})$. We prove that the gradient of $\left\{u_{n}\right\}$ is uniformly bounded. From standard compactness results, it will follow that $\left\{u_{n}\right\}$ contains a subsequence converging uniformly on compacts of $\Omega$ to a solution $u \in C^{2, \alpha}(\bar{\Omega})$ to $Q_{h}=0$ in $\Omega$ with $\left.u\right|_{\partial \Omega}=0$. It follows that $h \in T$ and $T$ is closed. Thus, $T=[0, H]$ proving Theorem 3 .

By contradiction, suppose the existence of $p_{n} \in \Omega$ such that $\lim _{n \rightarrow \infty}\left|\nabla u_{n}\right|$ $=\infty$. Without loss of generality, we may assume that $p_{n}$ converges to $p \in \bar{\Omega}$. From interior gradient estimates, $\left\{u_{n}\right\}$ contains a subsequence, which we consider as being $\left\{u_{n}\right\}$ itself, converging uniformly on compacts of $\Omega$ to a solution $u \in C^{2}(\Omega)$ to $Q_{h}=0$ in $\Omega$. This implies that $p \in \partial \Omega$. Let us consider the quarter of cylinder, say $C$, given by

$$
z(x, y)=\sqrt{\frac{1}{4 H^{2}}-x^{2}},-\frac{1}{2 H} \leq x \leq 0
$$

whose boundary consists of the straight lines $l_{1}:\{z=0, x=-1 /(2 H)\}$ and $l_{2}:\{z=1 /(2 H), x=0\}$. We apply a rigid motion on $C$ such that $l_{1}$ coincides with the tangent line to $\partial \Omega$ at $p$ and such that the projection of $l_{2}$ in the plane $z=0$ contains points of $\Omega$. Call this cylinder $C$ again. Moving $C$ slightly down, we have that the height of the line $l_{2}$, after this motion, is still bigger than $a$, and the norm of the gradient of $z(x, y)$ at $l_{1}$ is finite, say $D$. By the assumption on the sequence $\left\{u_{n}\right\}$, we can get $n$ such that $\left|\nabla u_{n}(p)\right|>D$. It follows that the graph $G_{n}$ of $u_{n}$ is locally above $C$ in a neighborhood of $p$. By hypothesis, the height of $G_{n}$ for any $n$ is smaller than $a$ so that, if we move $G_{n}$ vertically down we will obtain a last interior contact point between $G_{n}$ and $C$, a contradiction with the maximum principle, proving Theorem 3 .

Proof of Corollary 3. Assume that $\Omega$ is contained in the strip $\Lambda$ having as boundary two parallel lines $l_{1}$ and $l_{2}$ with

$$
d\left(l_{1}, l_{2}\right)=\inf \left\{\|x-y\| \mid x \in l_{1}, y \in l_{2}\right\} \leq \frac{1}{H}
$$


and let us first consider the case that $\Omega$ is compact. Given $l>0$, we consider the domain $\Lambda_{l}=\Lambda_{1} \cup \Lambda_{2} \cup \Lambda_{3}$ given by

$$
\begin{aligned}
& \Lambda_{1}=\left\{(x, y)|-l \leq x \leq l,| y \mid \leq \frac{1}{2 H}\right\} \\
& \Lambda_{2}=\left\{(x, y) \mid(x+l)^{2}+y^{2} \leq \frac{1}{4 H^{2}}, x \leq-l\right\} \\
& \Lambda_{3}=\left\{(x, y) \mid(x-l)^{2}+y^{2} \leq \frac{1}{4 H^{2}}, x \geq l\right\} .
\end{aligned}
$$

By choosing $l$ big enough, we have $\Omega \subset \Lambda_{l}$. It follows from to the proof of Theorem 3.2 of $[\mathbf{E F R}]$ the existence of a solution $v \in C^{2}\left(\Lambda_{l}\right) \cap C^{0}\left(\overline{\Lambda_{l}}\right)$ to $Q_{H}=0$ in $\Lambda_{l}$ such that $\left.v\right|_{\partial \Lambda_{l}}=0$ (obtained by using the method of Perron). Since on $\Lambda$ one can place a half cylinder of mean curvature $H$ whose boundary is the two lines $l_{1}$ and $l_{2}$, it follows immediately from the maximum principle that $a:=\max _{\Lambda_{l}} v<1 /(2 H)$. Using again the maximum principle, it follows that $\Omega$ satisfies the a priori height estimate $\max _{\Omega} u \leq a$ where $u \in C^{2}(\Omega) \cap C^{0}(\bar{\Omega})$ is any solution to $Q_{h}=0$ in $\Omega$ such that $\left.u\right|_{\partial \Omega}=0$, for any $h \in[0, H]$.

We consider now a sequence of convex bounded $C^{2, \alpha}$ domains $\Omega_{n}$ satisfying

$$
\Omega=\bigcup_{n=1}^{\infty} \Omega_{n}, \quad \overline{\Omega_{n}} \subset \Omega_{n+1} .
$$

Obviously the domains $\Omega_{n}$ satisfy the height estimate $\max _{\Omega_{n}} u \leq a$ so that we can get for any $n$, by Theorem 3 , a solution $u_{n} \in C^{2, \alpha}\left(\overline{\Omega_{n}}\right)$ to $Q_{H}=0$ in $\Omega_{n}$ with $\left.u_{n}\right|_{\partial \Omega_{n}}=0$. Standard compactness results guarantee us the existence of a subsequence of $\left\{u_{n}\right\}$, which we assume to be $\left\{u_{n}\right\}$ again, converging uniformly on compacts of $\Omega$ to a solution $u \in C^{2}(\Omega)$ to $Q_{H}=0$ in $\Omega$. By using quarter of cylinders as in the proof of Theorem 3 we have that the norm of the gradient of the family $\left\{u_{n}\right\}$ is uniformly bounded and therefore the gradient of $u$ is bounded on $\Omega$. It follows that $u \in C^{2}(\Omega) \cap C^{0}(\bar{\Omega})$ and $\left.u\right|_{\partial \Omega}=0$.

We suppose now that $\Omega$ is any convex domain in $\Lambda$ and set $\Omega_{n}=\Omega \cap \Lambda_{n}$. Choose $n_{0}$ such that $\Omega_{n}$ is non-empty for all $n \geq n_{0}$. Of course, $\left\{\Omega_{n}\right\}$ is a sequence of convex bounded domains contained in $\Omega$ satisfying the same conditions as above. According to what was proved before, there is, for each $n \geq n_{0}$ a solution $u_{n} \in C^{2}\left(\Omega_{n}\right) \cap C^{0}\left(\overline{\Omega_{n}}\right)$ to $Q_{H}=0$ in $\Omega_{n}$ such that $\left.u_{n}\right|_{\partial \Omega_{n}}=0$. Without loss of generality, we may assume that $\left\{u_{n}\right\}$ converges uniformly on compacts of $\Omega$ to a solution $u \in C^{2}(\Omega)$ to $Q_{H}=0$ in $\Omega$. To prove that $u \in C^{2}(\Omega) \cap C^{0}(\bar{\Omega})$ and $\left.u\right|_{\partial \Omega_{n}}=0$, in view that $\left.u_{n}\right|_{\partial \Omega_{n}}=0$, it is enough to prove that the family $\left\{u_{n}\right\}$ has uniformly bounded gradient norm in any compact $K$ of $\bar{\Omega}$. 
Let $K$ be a given compact of $\bar{\Omega}$. Let $n_{1}$ be such that $K \subset \Omega_{n}$ for all $n \geq n_{1}$. Clearly, one has $a:=\max _{\overline{\Omega_{n_{1}}}} u<1 /(2 H)$. Since, by the maximum principle, $u_{n} \leq u$ for all $n$, we have $\max _{\overline{\Omega_{n}}} u_{n} \leq a$, for all $n \geq n_{1}$. Hence, one can apply again the argument using quarters of cylinders to conclude that the family $\left\{u_{n}\right\}$ has uniformly bounded norm of the gradient in $\Omega_{n_{1}}$, and therefore in $K$, finishing the proof of Corollary 3.

Proof of Proposition 2. We have, since $|\nabla u|$ is not identicaly zero,

$$
\begin{aligned}
\operatorname{Area}(G) & =\int_{\Omega} \sqrt{1+|\nabla u|^{2}} d x<\int_{\Omega}\left(1+\frac{|\nabla u|^{2}}{\sqrt{1+|\nabla u|^{2}}}\right) d x \\
& =\operatorname{Area}(\Omega)+\int_{\Omega} \frac{|\nabla u|^{2}}{\sqrt{1+|\nabla u|^{2}}} d x .
\end{aligned}
$$

Given $s \in[0, h)$ we set

$$
\Omega_{s}=\left\{\left(x_{1}, x_{2}\right) \in \Omega \mid u\left(x_{1}, x_{2}\right) \geq s\right\}
$$

and $\Gamma_{s}=\partial \Omega_{s}$. By the coarea formula $([\mathbf{F}], 3.2 .22)$, one has

$$
\int_{\Omega} \frac{|\nabla u|^{2}}{\sqrt{1+|\nabla u|^{2}}} d x=\int_{0}^{h}\left[\int_{\Gamma_{s}} \frac{|\nabla u|}{\sqrt{1+|\nabla u|^{2}}} d l_{s}\right] d s .
$$

Integrating Equation (1) (with $\Omega_{s}$ in place of $\Omega$ ) and using divergence's theorem we obtain

$$
\int_{\Gamma_{s}} \frac{|\nabla u|}{\sqrt{1+|\nabla u|^{2}}} d l_{s}=2 H \operatorname{Area}\left(\Omega_{s}\right)
$$

so that

$$
\operatorname{Area}(G)<\operatorname{Area}(\Omega)+2 H \int_{0}^{h} \operatorname{Area}\left(\Omega_{s}\right) d s \leq(1+2 h H) \operatorname{Area}(\Omega),
$$

proving the first inequality of Proposition 2.

By Theorem 1 of $[\mathbf{L M}]$, we have

$$
\frac{2 \pi h}{H} \leq \operatorname{Area}(G)
$$

so that

$$
\frac{2 \pi h}{H}<(1+2 h H) \operatorname{Area}(\Omega)
$$

what gives the second inequality of Proposition 2, concluding its proof.

Proof of Corollary 5. It follows from the second inequality of Proposition 2 that given any solution $u \in C^{2}(\Omega) \cap C^{0}(\bar{\Omega})$ to $Q_{h}=0$ in $\Omega$ with $\left.u\right|_{\partial \Omega}=0$, $h \in[0, H]$ satisfies

$$
\max _{\Omega} u \leq a=\frac{\operatorname{Area}(\Omega) H}{2\left(\pi-H^{2} \operatorname{Area}(\Omega)\right)}
$$


and, since $2 \operatorname{Area}(\Omega) H^{2}<\pi$, it follows that $a<1 /(2 H)$. Corollary 5 therefore follows from Theorem 3 .

Proof of Theorem 4. Let $C_{1}, \ldots, C_{n}$ be the connected components of $\partial M$ and let $\Omega_{i}$ be the region enclosed by $C_{i}$. Anyone of the conditions (a), (b) or (c) guarantees that there is a graph $G_{i}$ over $\Omega_{i}$ with $\partial G_{i}=C_{i}$. We assume that $G_{i} \subset\{z \leq 0\}$, so that $N:=M \cup G, G=G_{1} \cup \ldots \cup G_{n}$ is a compact embedded topological surface without boundary which is $C^{\infty}$ differentiable with cmc $H$ in $N \backslash C, C=C_{1} \cup \ldots \cup C_{n}$. Furthermore, anyone of the conditions (a), (b) or (c) also implies that the tangent planes of $M$ and $G$ at a common point $p \in C$ form a inner angle (that is, respect to the normal $N=(1 / H) \vec{H}$ ) which is smaller than $\pi$ (see below).

We employ now the technique of Alexandrov to obtain a horizontal symmetry of $N$ (if $M$ is not a graph) considering a family of horizontal planes: Denote by $P_{t}$ the plane $\{z=t\}$. Set $U_{t}^{+}=\{z>t\}$ and $N_{t}^{+}=N \cap U_{t}^{+}$. Let $N_{t}^{*}$ be the reflection of $N_{t}^{+}$in the plane $P_{t}$. Let $V$ be the bounded connected component of $\mathbb{R}^{3} \backslash N$.

For $t$ big enough one has $U_{t}^{+} \cap N=\emptyset$. Set $t_{0}=\inf \left\{t \mid U_{t}^{+} \cap N=\emptyset\right\}$. For $t$ slightly smaller than $t_{0}$, we have $N_{t}^{*} \subset V$. Therefore, the number

$$
t_{1}=\inf \left\{t \leq t_{0} \mid N_{t}^{*} \subset V\right\}
$$

is well defined and satisfies $-\infty<t_{1}<t_{0}$. There are two possibilities: $t_{1} \leq 0$ or $t_{1}>0$. In the first one, it follows that $M$ is a graph over $P$. In the second one, $N_{t}^{-}:=N \backslash N_{t}^{+}$and $N_{t}^{*}$ are either tangent at the boundary or they have a common point, say $p$, belonging neither to the boundary of $N_{t}^{-}$nor to $N_{t}^{*}$. In the first case, it follows from the maximum principle at the boundary that $N_{t}^{-} \subset N_{t}^{*}$. It follows that $N_{t}^{+} \cup N_{t}^{*}$ is a compact embedded surface with cmc $H$ without boundary. By Alexandrov's theorem, $N_{t}^{+} \cup N_{t}^{*}$ is a sphere, and this proves Theorem 4 in this case. In the second case, $p$ cannot belong to $C$ because the plane tangent of $M$ and $G$ at $p$ form an acute angle. Therefore, $p$ is an interior regular point in $N_{t}^{*}$ and $N_{t}^{-}$so that $N_{t}^{*}$ and $N_{t}^{-}$are tangent at $p$. The maximum again implies that $N_{t}^{-} \subset N_{t}^{*}$ and this implies, as before, that $M$ is part of a sphere.

We now remark why conditions (a), (b) or (c) imply that the tangent planes form an acute angle as proclaimed above. Setting, as before, $N=$ $\left.(1 / H) \vec{H}\right|_{M}$, this is clearly the case if $\left\langle N, e_{3}\right\rangle<0$. Setting $N_{G}=\left.(1 / H) \vec{H}\right|_{G}$, one has therefore to prove that $\langle N(p), n(p)\rangle \geq\left\langle N_{G}(p), n(p)\right\rangle$ if $\left\langle N(p), e_{3}\right\rangle \geq$ 0 , where $p \in \partial M$ and $n$ is the unit normal vector field along $\partial M$ in the plane $P$, orthogonal to $\partial M$.

From the hypothesis, in case (a), it follows that any point $p$ in a connected component $C$ of $\partial M$ belongs to a circle $L \subset P$ of radius $R_{0}=1 / \kappa_{0}$ tangent to $C$ at $p$ and whose interior contains the interior of $C$. By the maximum principle, we have $\left\langle N_{G}, n\right\rangle \leq\left\langle N_{S}, n\right\rangle$, where $S$ is the graph a cup 
sphere with cmc $H$ such that $\partial S=L$. Now, direct computations show that $\left\langle N_{S}, n\right\rangle=H / \kappa_{0}$, proving our claim in this case.

In case (b), since the convex domain $\Omega$ enclosed by $C$ is between two parallel lines $l_{1}, l_{2}$ with $d\left(l_{1}, l_{2}\right)=d$, considering the part of cylinder of cmc $H$ having as boundary $l_{1}$ and $l_{2}$, we conclude that the height of $G$ is at most

$$
\frac{1-\sqrt{1-d^{2} H^{2}}}{2 H} \text {. }
$$

Therefore one can use, up to congruencies, the part of cylinder

$$
v(x, y)=\sqrt{\frac{1}{4 H^{2}}-x^{2}}-\frac{\sqrt{1-d^{2} H^{2}}}{2 H}, \quad-\frac{d}{2} \leq x \leq 0
$$

as a barrier at any point of $C$ so that we will have $\left\langle N_{G}, n\right\rangle \geq\left\langle N_{S}, n\right\rangle$, where $S$ now is the graph of $v$ in the given domain. A computation then shows that

$$
\left\langle N_{S}, n\right\rangle=\frac{d H}{\sqrt{1-d^{2} H^{2}}},
$$

proving our claim in the case (b).

In case (c), we have that the height of $G$ (see the proof of Corollary 5 ) is at most

$$
\frac{A H}{2\left(\pi-H^{2} A\right)}
$$

so that we can use the some reasoning of case (b) to conclude that

$$
\left\langle N_{S}, n\right\rangle=\frac{\sqrt{H^{2} A\left(2 \pi-3 H^{2} A\right)}}{\pi-2 H^{2} A}
$$

finishing the proof of Theorem 4 .

Proof of Corollary 6. We first observe that the conditions $2 a H^{2} \leq \pi$ and (6) imply that $A H^{2} \leq 2 \pi$ so that, by Corollary 3 of $[\mathbf{L M}], M$ is contained in the half space $z \geq 0$. We prove that the condition

$$
\langle N(p), n(p)\rangle \geq \frac{H \sqrt{a\left(2 \pi-3 H^{2} a\right)}}{\pi-2 H^{2} a}
$$

if $\left\langle N(p), e_{3}\right\rangle \geq 0, p \in \partial M$, is satisfied (we are using the same notations of Theorem 4). For, choose a point $p \in \partial M$ where $\left\langle N(p), e_{3}\right\rangle \geq 0$. Using reflections on vertical planes orthogonal to $n(p)$ and the maximum principle, we may conclude that $G_{p}$ is a graph over $P$, where $P$ is the plane orthogonal to $n(p)$ through $p$ and $G_{p}$ the closure of the connected component of $\left(\mathbb{R}^{3} \backslash P\right) \cap$ $M$ not containing $\partial M$. It is clear that the area $a_{p}$ of $G_{p}$ is smaller than $A-a$ and we may apply Theorem 1 of $[\mathbf{L M}]$ to conclude that

$$
h_{p} \leq \frac{H a_{p}}{2 \pi}<\frac{H(A-a)}{2 \pi},
$$


where $h_{p}$ is the height of $G_{p}$. Let us assume that $G_{p}$ is given as the graph of a function $u_{p} \in C^{2}\left(\overline{\Lambda_{p}}\right)$ where $\Lambda_{p}$ is some domain in the plane $P$. Then (11) is implied by the inequality

$$
\left|\nabla u_{p}(p)\right| \leq \frac{H \sqrt{a\left(2 \pi-3 H^{2} a\right)}}{\pi-2 H^{2} a} .
$$

Observe that $\Lambda_{p}$ is globally convex at $p$ and it follows from (6), (12) and the condition $2 a H^{2} \leq \pi$ that

$$
\frac{H(A-a)}{2 \pi}<\frac{1}{2 H}
$$

By the maximum principle we may conclude that $G_{p}$ is below the piece $C_{p}$ of a cmc $H$ cylinder given as a graph over the plane $P$ having as boundary the straight tangent line $t$ to $\partial \Lambda_{p}$ at $p$ and a straight line $s$ above $P$ parallel to $P$ and $t$ at a height

$$
\frac{H(A-a)}{2 \pi}
$$

from $P$. Hence we can estimate the norm of the gradient of $u_{p}$ at $p$ by the gradient of $C_{p}$ at $t$ to obtain

$$
\left|\nabla u_{p}(p)\right| \leq \frac{\sqrt{\frac{H(A-a)}{2 \pi}\left(\frac{1}{H}-\frac{H(A-a)}{2 \pi}\right)}}{\frac{1}{2 H}-\frac{H(A-a)}{2 \pi}} .
$$

It follows from (6) that

$$
\frac{\sqrt{\frac{H(A-a)}{2 \pi}\left(\frac{1}{H}-\frac{H(A-a)}{2 \pi}\right)}}{\frac{1}{2 H}-\frac{H(A-a)}{2 \pi}} \leq \frac{H \sqrt{a\left(2 \pi-3 H^{2} a\right)}}{\pi-2 H^{2} a}
$$

so that (13) and therefore (11) is satisfied. Corollary 6 then follows from Theorem 4, observing that condition (6) is never satisfied by a large cap sphere.

Theorem 5. Let $M$ be a compact embedded surface with cmc $H=1$ in $\mathbb{R}^{3}$, with boundary $\partial M$ contained in $\mathbb{R}_{+}^{3}=\{z \geq 0\}$ and having a $1-1$ projection over a closed $C^{2, \alpha}$ a convex curve $\gamma$ on the plane $P=\{z=0\}$. Set $C:=\gamma \times \mathbb{R}$ (the right cylinder over $\gamma$ ), $\Omega:=\operatorname{int}(\gamma)$, and let $C_{-}$be the connected component of $C \backslash \partial M$ which is below $\partial M$, that is, containing points with $z<0$. We require that

(a) there exists a neighborhood $U$ of $\partial M$ in $M$ which is a graph over a neighborhood $\Lambda \subset \bar{\Omega}$ of $\partial \Omega$;

(b) $M \cap C_{-}=\emptyset$.

Then $M$ is a graph over $\bar{\Omega}$. 
Proof. We claim that, up to a reflection on the plane $z=0$ followed by a translation along the $z$-axis, one can assume that the mean curvature vector of $U$ points towards the plane $z=0$. In fact, if the mean curvature vector points to the other direction, let us consider the topological compact (closed) surface

$$
K=M \cup T \cup \bar{\Omega},
$$

where $T:=\{z \geq 0\} \cap C_{-}$. Since $M \cap C_{-}=\emptyset, K$ is embedded. Therefore the mean curvature vector of $M$ points to the unbounded component of $\mathbb{R}^{3} \backslash K$. By the maximum principle, any plane coming from the infinity, approaching $\partial M$, and not intersecting $C_{-}$, can not intersect $M \backslash \partial M$. It follows that $M \cap$ $\left(C \backslash C_{-}\right)=\emptyset$. Therefore, reflecting $M$ on the plane $z=0$ and applying a translation along the $z$ - axis, the new surface satisfies our claim.

Let us assume that $U$ is the graph of a function $v \in C^{2}(\bar{\Lambda} \backslash \gamma) \cap C^{0}(\bar{\Lambda})$. Let $\Omega_{0} \subset \Omega$ be a subdomain with smooth boundary close enough to $\Omega$ so that $\partial \Omega_{0} \subset \Lambda \backslash \gamma$ and such that $\partial \Omega_{0}$ is $C^{2, \alpha}$ and strictly convex.

We prove now the existence of a solution to Dirichlet's problem for $\mathrm{cmc}$ 1 graphs:

$$
\operatorname{div} \frac{\nabla u}{\sqrt{1+|\nabla u|^{2}}}=-2, \quad u \in C^{2}\left(\bar{\Omega}_{0}\right),\left.\quad u\right|_{\partial \Omega_{0}}=\phi
$$

where $\phi:=\left.v\right|_{\partial \Omega_{0}}$. For, we consider a continuous homotopy between (14) and the Dirichlet problem for the minimal surface equation given by

$$
\operatorname{div} \frac{\nabla u}{\sqrt{1+|\nabla u|^{2}}}=-2 H, \quad u \in C^{2}\left(\bar{\Omega}_{0}\right),\left.\quad u\right|_{\partial \Omega_{0}}=\phi,
$$

$H \in[0,1]$. The classical theorem of T. Radó about existence of a solution to the Dirichlet's problem for the minimal surface equation in convex domains guarantees the existence of a solution of (15) for $H=0$. It follows from the inverse function theorem, that (15) has a solution for $0 \leq H<H_{1}$. To guarantee that (15) has a solution with $H=1$, we prove that any solution of (15) for $H_{1} \leq H \leq 1$ has a priori $C^{1}$ bound estimates in the whole domain $\Omega_{0}$. Thus, choose $H$ with $H_{1} \leq H \leq 1$ and let $u$ be a solution of (15). As it is well known, one has

$$
|u| \leq \frac{1}{H_{1}}+\max _{\partial \Omega_{0}} \phi
$$

Observe that orientation of the graph $G$ of $u$ is such that

$$
\vec{H}=\frac{H(\nabla u,-1)}{\sqrt{1+|\nabla u|^{2}}},
$$

where $\vec{H}$ is the mean curvature vector of $G$. Therefore, if $n_{1}$ denotes the interior normal vector to $\partial \Omega_{0}$, then $\left\langle\nabla u, n_{1}\right\rangle=\sqrt{1+|\nabla u|^{2}}\left\langle\vec{H}, n_{2}\right\rangle$, where 
$n_{2}$ is the interior unit normal vector to the cylinder $D$ over $\partial \Omega_{0}$ at $\partial G$. Therefore, if there is $p_{0} \in \gamma$ such that

$$
\left\langle\nabla u(p), n_{1}(p)\right\rangle \rightarrow-\infty
$$

as $p \rightarrow p_{0}, p \in \Omega_{0}$, then

$$
\left\langle\vec{H}(p), n_{2}(p)\right\rangle \rightarrow-1
$$

as $p \rightarrow p_{0}$. We observe that this possibility can not happen: Since $\Omega_{0}$ is strictly convex one can place, at any point $q$ in the boundary of the graph $G$, a circular cylinder $E$ of constant mean curvature $H$ tangent to the cylinder $D$ over $\partial \Omega_{0}$, so that $C \cap D$ is a straight vertical line. Moreover, $\Omega_{0}$ and $\phi$ satisfy the bounded slope condition (see $[\mathbf{G T}]$, Remark 4, p. 255). Therefore, we can slightly tilt $E$, fixing $q$, so that it still intersects the boundary of $G$ only at $q$. Hence, by the maximum principle, one gets a barrier at any point of the boundary which provides a uniform bound from below for $\left\langle\nabla u, n_{1}\right\rangle$. We prove now that one can uniformly estimate this quantity from above.

Since $\partial \Omega_{0} \subset \Omega$ and $d\left(\partial \Omega_{0}, \partial \Omega\right)>0$, it follows from gradient interior bound estimates that $|\nabla v|$ is uniformly bounded on $\partial \Omega_{0} \cap \Lambda$. Therefore, since the maximum of $|\nabla u|$ in $\Omega_{0}$ is assumed in $\partial \Omega_{0}$, it is enough to prove that

$$
\left\langle\nabla u(p), n_{1}\right\rangle \leq\left\langle\nabla v(p), n_{1}\right\rangle
$$

for all $p \in \partial \Omega_{0}$. By contradiction, assume that

$$
\left\langle\nabla u(p), n_{1}\right\rangle>\left\langle\nabla v(p), n_{1}\right\rangle
$$

at some $p \in \partial \Omega_{0}$. From (17), it follows that $M$ has points below $G$ near $p$. Therefore, by moving $G$ slightly down, we then have that $\partial G \cap M=\emptyset$ while $G \cap M \neq \emptyset$. Therefore, by moving $G$ down until it reaches the last point of contact with $M$, since $M \cap C_{0_{-}}=\emptyset$, we obtain a tangency between $M$ and $G$ at a interior point of both $G$ and $M$, a contradiction, according to the maximum principle. Hence, any solution $u$ of (15) satisfies (16). Therefore, using bound interior estimates for the gradient, it follows that any solution of (15) is uniformly bounded in modulus by above and has uniformly bounded gradient. Therefore, (10) admits a solution $u \in C^{2}\left(\bar{\Omega}_{0}\right)$ for $H=1$. Let $G$ be the graph of $u$.

Denote by $\lambda$ and by $\eta$ the interior conormal to $\partial G$ and $\partial M^{\prime}\left(\partial G=\partial M^{\prime}\right)$, respectively, where $M^{\prime}=M \backslash \operatorname{graph}\left(\left.v\right|_{\left(\Omega \backslash \Omega_{0}\right)}\right)$. We note that, as we have explained in the first paragraph of this proof, $M$ and $G$ induce the same orientation on their common boundary. By (16), we have

$$
\left\langle\eta(p), e_{3}\right\rangle \geq\left\langle\lambda(p), e_{3}\right\rangle
$$

for all $p \in \partial M^{\prime}$. On the other hand, if one considers a smooth surface $T$ with boundary $\partial T=\partial M^{\prime}$ such that $M^{\prime} \cup T$ and $G \cup T$ are immersed two cycles, 
and if $\rho$ denotes the unit normal to $T$ pointing to the bounded component of $\mathbb{R}^{3} \backslash(G \cup T)$, then the balancing formula (Proposition I.1.8 of $[\mathbf{K}]$ ) gives

$$
\int_{\partial M^{\prime}} \eta=-H \int_{T} \rho=\int_{\partial G} \lambda
$$

This implies that

$$
\left\langle\eta(p), e_{3}\right\rangle=\left\langle\lambda(p), e_{3}\right\rangle
$$

for $p \in \partial M^{\prime}$ so that $G$ and $M^{\prime}$ are tangent at the boundary. The maximum principle therefore implies that $M^{\prime}=G$ and the theorem is proved.

Assuming that $\partial M$ is a plane curve, we can prove, using the same technique of Theorem 5 , that $M$ is a graph, with weaker hypothesis than of Theorem 5, namely:

Theorem 6. Let $M$ be a compact embedded cmc $H \geq 0$ surface whose boundary is a planar curve in the plane $z=0$, and assume the existence of a neighborhood $U$ of $\partial M$ in $M$ lying in $\{z \geq 0\}$ which is a graph over the plane $z=0$. Then $M$ is a graph over $z=0$.

Proof. Of course, if $H=0$ then the result is trivial, so that let us assume $H>0$. We first show that the mean curvature vector of $U$ points to the plane $z=0$. Consider the immersed surface $K=M \cup \Omega$, where $\Omega$ is the planar domain bounded by $\partial M$. We choose a unit normal vector $N$ to $K$ in $K \backslash \partial M$, respecting the orientation of $K$, such that $\left.N\right|_{M}=(1 / H) \vec{H}$, where $\vec{H}$ is the mean curvature vector of $M$. At $\Omega$, one has $N= \pm(0,0,1)$. Therefore, our claim is proved if we show that $\left.N\right|_{\Omega}=e_{3}=(0,0,1)$.

It follows from Proposition I.1.8 of $[\mathbf{K}]$ that

$$
\int_{\partial M}\left\langle v, e_{3}\right\rangle d s=H \int_{\Omega}\left\langle N, e_{3}\right\rangle d A
$$

where $v$ is the unit interior conormal vector to $M$ at $\partial M$. Since $U$ lies in $\{z \geq 0\}$ we necessarily have $\left\langle v, e_{3}\right\rangle \geq 0$. Since $H>0$ we obtain $\left.N\right|_{\Omega}=e_{3}$.

By the maximum principle, it follows that $U$ can not be tangent to $z=0$ along $\partial M$. It follows from Theorem 2 of [BEMR] that $M$ lies in $z \geq 0$. The proof now goes exactly as in Theorem 5: Using the continuity method we prove the existence of cmc $H$ graph $G$ defined in $\Omega$ and vanishing at $\partial \Omega$. Using again the balancing formula, we may therefore conclude that $M=G$, proving Theorem 6 .

\section{References}

[BEMR] F. Brito, R. Earp, W. Meeks and H. Rosenberg, Structure theorems for constant mean curvature surfaces bounded by a planar curve, Indiana Univ. Math. Journal, 40 (1991), 333-343. 
[doCD] M.P. do Carmo and M. Dajczer, Rotational hypersurfaces in spaces of constant curvature, Transactions of the A.M.S., 2 (1983), 277.

[CK] P. Collin and R. Krust, Le problème de Dirichlet pour l'équation des surfaces minimales sur de domaines non bornés, Bull. Soc. Math. de France, 119 (1991), 443-462.

[ER] R.S. Earp and H. Rosenberg, The Dirichlet problem for the minimal surface equation on unbounded planar domains, Journal des Math. Pures et Apliquées, 68 (1989), 163-183.

[ET] R.S. Earp and E. Toubiana, Some applications of maximum principle to hypersurface theory in euclidean and hyperbolic space, preprint.

[EFR] N. do Espírito-Santo, K. Frensel and J. Ripoll, Characterization and existence results on $H$-domains, preprint.

[F] H. Federer, Geometric Measure Theory, Springer-Verlag, New York, 1969.

[GT] D. Gilbarg and N.S. Trudinger, Elliptic Partial Differential Equations of Second Order, 2nd edition, Springer-Verlag, 1983.

[K] R.B. Kusner, Global geometry of extremal surfaces in three space, Dissertation at University of California, Berkeley.

[KT] N. Kutev and F. Tomi, Existence and Nonexistence in the Exterior Dirichlet Problem for the Minimal Surface Equation in the Plane, to appear in the Journal of Differential and Integral Equations.

[LM] R. López and S. Montiel, Constant mean curvature surfaces with planar boundary, Duke Math. Journal, 85(3) 1996, 583-605.

[N] J.C.C. Nitsche, Lectures on Minimal Surfaces, Vol. I, Cambridge University Press, 1989.

[PP] L.E. Payne and G.A. Philippin, Some maximum principles for nonlinear elliptic equations, Nonlinear Analysis, Theory, Methods and Applications, 3(2) (1979), 193-211.

[R] J. Ripoll, Some existence and gradient estimates of solutions of the Dirichlet problem for the constant mean curvature equation in convex domains, to appear in the J. of Diff. Equations.

[RT] J. Ripoll and F. Tomi, Some existence theorems for minimal graphs over nonconvex planar domains, preprint.

[RR] A. Ros and H. Rosenberg, Constant mean curvature surfaces in a half-space of $\mathbb{R}^{3}$ with boundary in the boundary of a half-space, Journal of Differential Geometry, 44(4) (1996), 807-817.

[S] J. Serrin, The Dirichlet problem for surfaces of constant mean curvature, Proc. of London Math. Society, 21(3) (1970), 361-384.

Received February 19, 1999. The author was partially supported by CNPq (Brazil).

Universidade Federal do R. G. Do Sul

Instituto DE MATEMÁtica

Av. Bento Gonçalves 9500

90540-000 Porto Alegre RS

BRAZIL

E-mail address: ripoll@mat.ufrgs.br 